\title{
Comparison of Quality of Life in Breast Cancer Survivors with Lymphoma Survivors
}

\author{
Mumtaz Ahmad Khan ${ }^{1}$, Shaista Zafar' ${ }^{2}$, Namrah Mahmood ${ }^{3}$ \\ ${ }^{1}$ Associate Professor, Department of General Surgery, PIMS, Islamabad Pakistan \\ ${ }^{2}$ Senior Registrar, Department of General Surgery, PIMS, Islamabad Pakistan \\ ${ }^{3}$ Medical Officer, Department of General Surgery, PIMS, Islamabad Pakistan
}

\begin{abstract}
A B S TR ACT
Background: Breast cancer diagnosis and its subsequent treatment, especially surgical removal of the breast(s) cause changes in physical appearance that can be devastating to the Quality of Life $(Q O L)$ of cancer survivors. The objective of this study was to assess the effect of surgical intervention on the QOL of breast cancer survivors by comparing it with QOL of Lymphoma survivors.

Material and Methods: This was a cross-sectional study, conducted in the Departments of General Surgery and Oncology at Pakistan Institute of Medical Sciences (PIMS) Islamabad, Pakistan from 1st June to 31st December, 2019. Six months' post treatment patients of breast cancer $(n=166)$ and lymphoma $(n=50)$ were included. A standardized questionnaire was used to assess the Quality of Life in Cancer Survivors (QOL-CSV). Chi square and independent t-test were applied to determine the association of QOL among lymphoma and breast cancer survivors. Linear regression model was applied to determine the confounding variables.

Results: All participants responded moderately in reporting overall quality of life in lymphoma survivors (LS) as compared to Breast cancer survivors (BCS) group (100\% vs $86.7 \%$; $P=.007)$. Physical wellbeing parameters revealed a better mean score of $75.86 \pm 10.53$ for LS group than the BCS group $(P<.001)$. Psychological wellbeing mean score was similar for both groups $(P=.46)$. Distress associated with illness and treatment was dealt better by LS than BCS group $(P<.001)$, whereas spiritual wellbeing was markedly better in BCS group $(P<.001)$. Fear of recurrence and spread was poor amongst the BCS than LS, while the LS group performed worse in social factors (mean value of $26.88 \pm 6.67)(P<$ .001).

Conclusions: Both lymphoma and breast cancer negatively affect the quality of life of the patients, especially the psychological, social and spiritual wellbeing. However, surgical intervention in breast cancer patients led to considerable worsening of the quality of life with respect to physical and social wellbeing and increased distress of illness and fear factor.

Key words: Breast cancer survivors, Lymphoma survivors, Psychological wellbeing, Quality of life, Spiritual wellbeing, Surgical intervention.

Authors' Contribution:
${ }^{1}$ Conception; Literature research;
manuscript design and drafting; ${ }^{2,3}$ Critical
analysis and manuscript review; Data
analysis; Manuscript Editing.

Article info:

Received: August 18, 2020

Accepted: December 17, 2020

Namrah Mahmood

Email:dr.namrahmahmood@gmail.com
\end{abstract}

Cite this article. Khan MA, Zafar S, Mahmood N. Comparison of Quality of Life in Breast Cancer Survivors with Lymphoma Survivors. J Islamabad Med Dental Coll. 2020; 9(4): 262-268. Doi: 10.35787/jimdc.v9i4.587
Funding Source: Nil Conflict of Interest: Nil 


\section{Introduction}

There is a surge in the cases of cancer globally with national indices following this trend. Pakistan reported 148,000 new carcinoma cases in 2012. ${ }^{1}$ Early detection and better treatment options have led to improvement in the survival rates of cancer patients worldwide. ${ }^{2}$ This has caused an increased interest in assessing the quality of life (QOL) of cancer survivors, based on the Bio-Psycho-Social model of health care. ${ }^{3}$ There is an overall rise in the global and national incidence of lymphoma, with a reported incidence of 8.4 per 100,000 in males and 6.5 per 100,000 in female population in Pakistan. ${ }^{4}$ Similarly, the incidence of breast cancer has also increased globally, with Pakistan reporting 50 per 100,000 of female population affected by breast cancer. ${ }^{5}$ The mainstay of treatment in lymphoma patients is chemotherapy with or without radiotherapy ${ }^{6}$ However, in the treatment of breast carcinoma, different surgical options are considered, based on the underlying pathology, along with chemotherapy and/or radiotherapy. ${ }^{7}$

The diagnosis of cancer and its subsequent treatment impairs the quality of life of the affected individual in multiple ways causing altered pain threshold, fatigue, sleeplessness and fear of future diagnoses. ${ }^{8}$ However, surgical treatment especially breast surgeries, may further impair quality of life with respect to body image and personal outlook. Social stigmas and concerns may further add to the distress of the patients. ${ }^{9}$ Since mastectomy involves removal of breast, which defines female appearance and contribute to sexuality, it can have a profound impact on the QOL of the patient in the long run. ${ }^{10}$

The main aim of this study was to understand the effect of surgical intervention (i.e. mastectomy) on the quality of life of breast cancer survivors'. However, cancer diagnosis, fear of recurrence, lengthy and costly treatment options as well as the side effects of chemotherapy are multiple factors which can also contribute in deranging quality of life, making it difficult to study the isolated effect of mastectomy on QOL. For this purpose, comparison is made with lymphoma survivors in this research in order to find the effect of mastectomy on the QOL of the patients.

\section{Material and Methods}

This cross-sectional study was conducted at the Outpatient Department of General Surgery and Oncology at PIMS, Islamabad Pakistan, from $1^{\text {st }}$ June 2019 to $31^{\text {st }}$ December 2019. Patients (age $\geq 18$ years) who had undergone mastectomy (Modified Radical Mastectomy) for breast cancer and had received treatment (chemotherapy and/ or radiotherapy) more than 6 months ago were included in the Breast Cancer Survivors (BCS) group. Whereas adult lymphoma patients (age $\geq 18$ years) who had received chemotherapy and/ or radiotherapy at least 6 months ago were included in the Lymphoma Survivors (LS) group. Patients who had residual disease, recurrence of disease or complications after treatment (related to surgery, chemotherapy or radiotherapy), and patients who had a previous history of any type of cancer, were excluded from this study. Sample size for each group (BCS and LS) was calculated using national prevalence of breast cancer and lymphoma. ${ }^{4,5}$ Non probability consecutive sampling technique was employed to recruit participants.

Permission to conduct this study was obtained from the Ethical Review Board of Shaheed Zulfiqar Ali Bhutto Medical University, Islamabad Pakistan. A written informed consent was taken from the participants and their demographical variables including age, profession, gender, type of carcinoma, stage of disease at diagnosis, type of 
treatment received and time since treatment ended was recorded. The principal investigators interviewed the participants and filled in the questionnaires. A standardized questionnaire (QOL$\mathrm{CSV}^{4}$ ) was used to assess the quality of life of the participants, with questions assessing physical, psychological, social and spiritual wellbeing as well as fear factor and distress of illness.

Data was entered and analyzed using SPSS version 20. Comparison of the scores of both groups was made using mean values for each category (physical, psychological, distress of illness, fear factor, social and spiritual wellbeing). Each of the 41 questions in QOL-CSV had values ranging from ' 0 ' to ' 10 ' with ' 0 ' as worst outcome and ' 10 ' as best outcome. Scores for each category were analyzed into poor, moderate and good with cut-off value kept at $<40 \%$, $41-70 \%$ and $>70 \%$ of the total score for each category, respectively. Frequencies and percentages were reported for categorical data and mean \pm standard deviation was recorded for numerical data. Chi square test and independent t-test was applied to determine the association of quality of life among lymphoma and breast cancer survivors for categorical and numerical variables, respectively. Linear regression model was applied to determine the confounder variables. $P$-value was considered as significant at $<.05$.

\section{Results}

A total of 50 Lymphoma survivors (LS) and 166 Breast cancer survivors (BCS) participated in the study, with a mean age of $55.84 \pm 8.82$ years in lymphoma group and $54.65 \pm 6.291$ years in breast cancer group, respectively.

In the LS group, a total of 24 (48\%) female and 26 (52\%) male patients were included, while in the BCS group, all participants were female. Most of the participants were married in both groups (LS 96\%; BCS 97.6\%). Regarding professions, all the BCS were housewives $(100 \%)$, while $54 \%$ of LS were housewives followed by $24 \%$ in-service, $10 \%$ businessmen, and $12 \%$ unemployed, respectively. Duration of treatment and stage of cancer at presentation in both groups are presented in Figures $1 \mathrm{~A}$ and $1 \mathrm{~B}$, respectively.

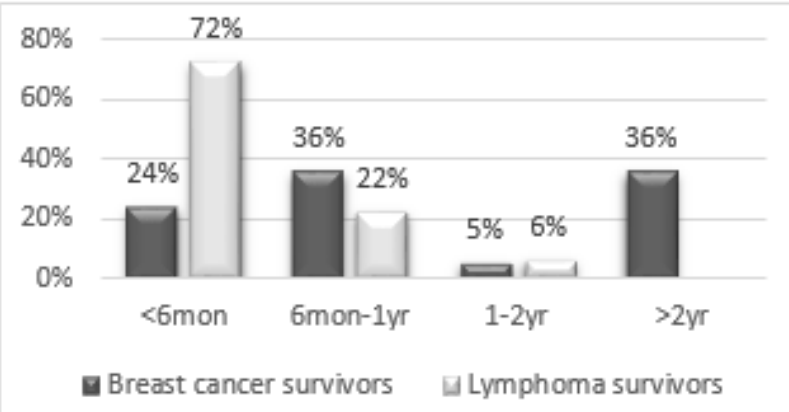

Figure 1A: Duration of treatment among both groups

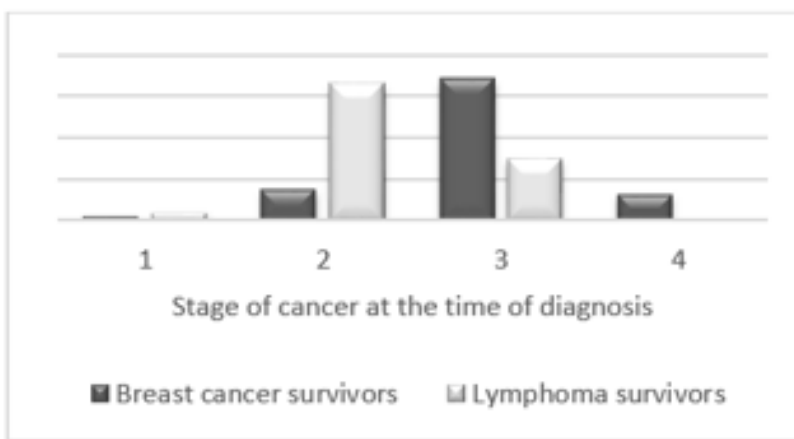

Figure 1B: Stage of cancer at the time of diagnosis

All participants responded moderately in reporting overall quality of life in LS group as compared to BCS group (100\% vs $86.7 \% ; P=.007$ ). We found significant association among $\mathrm{BCS}$ group and LS group in overall comparison of QOL $(P<.001)$ (Table I). In Physical wellbeing, LS group took the lead with $55 \%$ of the participants scoring more than 80 out of 90 score and a mean value of $75.86 \pm 10.53$ as compared to BCS group $(P<.001)$. Breast cancer survivors reported higher incidence of bodyaches, fatigue and menstrual problems (74\% participants scoring less than 5 out of 10 in each question). Both groups performed moderately (41\%-70\%) in Psychological wellbeing, with mean value of $42.18 \pm 8.06$ and 43.04 \pm 7.04 in LS and BCS groups, respectively $(P=.46)$. However, the responses about changes in appearance and self-concept revealed that more 
than $80 \%$ of breast cancer survivors reported extreme changes (scoring $\geq 8$ out of 10 ) (Table I).

There was significant correlation in quality of life amongst LS and BCS for physical wellbeing, distress of illness and treatment, extent of fearfulness, social concerns, spiritual wellbeing $(P<0.001)$ (Table I). However, no significant correlation was found in psychological wellbeing between both groups.

Distress of illness and treatment showed that majority ( $56 \%$ vs $76 \%$ ) were reported moderately (QOL score $41 \%-71 \%$ ) in BCS and LS groups, respectively with mean value of $40.84 \pm 6.17$ vs $36.86 \pm 6.55 \quad(P<.001)$. Among extent of fear questions, fear of recurrence and second cancer was poor in BCS group as shown by less score in QOL for most of the participants (72.3\%) as compared to LS group (50\%). Quality of Life regarding social concerns amongst LS group was poorer than with BCS group. However, in BCS group more participants reportedly felt that their sexuality has been negatively affected (64\% participants scored more than 5 out of 10). Spiritual wellbeing amongst LS was better as compared to BCS as shown in Table II.

Age, sex, marital status, duration of treatment, stage of cancer, profession type and treatment type showed significant association with overall quality of life in $B C S$ group as compared to $L S$ group $(R=0.276$; adjusted R-squared 4.7\%; $P=.026$ vs $R=0.416$; adjusted R-squared 5.8\%; $P=.201$ ) in linear regression model. Marital status and stage of cancer were the contributing factors in developing poor QOL in BCS (Table III).

\begin{tabular}{|l|c|c|c|}
\hline \multicolumn{4}{|c|}{ Table I: Comparison of mean score of Quality of life between Breast cancer survivors and Lymphoma survivors } \\
\hline Quality of Life & BCS (Mean \pm SD) & LS (Mean \pm SD) & $\boldsymbol{P}_{\text {-value }}{ }^{*}$ \\
\hline Overall Score & $48.25 \pm 6.94$ & $55.27 \pm 4.31$ & $<.001$ \\
\hline Physical well being & $17.09 \pm 7.33$ & $75.86 \pm 10.53$ & $<.001$ \\
\hline Psychological well being & $43.04 \pm 7.04$ & $42.18 \pm 8.06$ & .461 \\
\hline Distress of illness and treatment & $36.86 \pm 6.55$ & $40.84 \pm 6.17$ & $<.001$ \\
\hline Fearfulness & $11.28 \pm 8.2$ & $16.58 \pm 4.30$ & $<.001$ \\
\hline Social concerns & $42.57 \pm 8.43$ & $26.88 \pm 6.67$ & $<.001$ \\
\hline Spiritual well being & $47.15 \pm 6.27$ & $30.64 \pm 6.83$ & $<.001$ \\
\hline
\end{tabular}

* Independent t-test was applied to determine the $P$-value considered significant at $<.05$

\begin{tabular}{|c|c|c|c|c|c|}
\hline \multicolumn{6}{|c|}{ Table II: Quality of life categories between Breast cancer survivors and Lymphoma survivors } \\
\hline & & Poor $\mathrm{n}(\%)$ & Moderate $\mathrm{n}(\%)$ & Good n (\%) & P-value ${ }^{*}$ \\
\hline \multirow{2}{*}{ Physical Well Being } & BCS & $166(100)$ & 0 & 0 & \multirow{2}{*}{$<.001$} \\
\hline & LS & 0 & $6(12)$ & $44(88)$ & \\
\hline \multirow{2}{*}{ Psychological Well Being } & BCS & $18(10.8)$ & $148(89.2)$ & 0 & \multirow{2}{*}{.326} \\
\hline & LS & $8(16)$ & $42(84)$ & 0 & \\
\hline \multirow{2}{*}{ Distress of Illness and Treatment } & BCS & $73(44)$ & $93(56)$ & 0 & \multirow{2}{*}{.011} \\
\hline & $\mathrm{LS}$ & $12(24)$ & $38(76)$ & 0 & \\
\hline \multirow{2}{*}{ Extent of Fearfulness } & BCS & $120(72.3)$ & $44(26.5)$ & $2(1.2)$ & \multirow{2}{*}{.006} \\
\hline & LS & $25(50)$ & $25(50)$ & 0 & \\
\hline \multirow{2}{*}{ Social Concerns } & BCS & $25(15.1)$ & $135(81.3)$ & $6(3.6)$ & \multirow{2}{*}{$<.001$} \\
\hline & LS & $39(78)$ & $11(22)$ & 0 & \\
\hline \multirow{2}{*}{ Spiritual Wellbeing } & BCS & $1(0.6)$ & $103(62)$ & $62(37.3)$ & \multirow{2}{*}{$<.001$} \\
\hline & LS & $18(36)$ & $32(64)$ & 0 & \\
\hline
\end{tabular}

* Chi square test was applied to determine the $P$-value considered significant at $<.05$ BCS-Breast cancer survivors; LS-Lymphoma survivors 


\begin{tabular}{|l|c|c|c|c|}
\hline \multirow{2}{*}{ Variables } & \multicolumn{2}{|c|}{ Table III: Comparison of Bio data variables in both groups } \\
\cline { 2 - 5 } & B (Cl 95\%) & P-value ${ }^{*}$ & \multicolumn{2}{c|}{ Lymphoma survivors } \\
\hline Age & $0.095(-0.10-0.29)$ & .338 & $0.107(-0.35-0.25)$ & $\boldsymbol{P}_{\text {-value }}{ }^{*}$ \\
\hline Sex & - & - & $-0.076(-2.67-2.52)$ & .137 \\
\hline Marital Status & $14.31(3.7-24.9)$ & .008 & $4.67(-1.9-11.2)$ & .953 \\
\hline Treatment Duration & $-1.05(-2.03--0.08)$ & .033 & $-1.59(-3.66-0.49)$ & .159 \\
\hline Stage of cancer & $1.2(-0.68-3.11)$ & .209 & $-0.51(-2.95-1.92)$ & .130 \\
\hline Profession Type & - & - & $-0.85(-2.04-0.34)$ & .672 \\
\hline Treatment Type & $0.57(-0.48-1.63)$ & .285 & - & .157 \\
\hline
\end{tabular}

${ }^{*} P<.05$ was considered statistically significant

\section{Discussion}

In this study all the participants in BCS group were females. Incidence of breast-related diseases in men is uncommon, with a prevalence ratio ranging from 0.1 to 3.4 cases per 100,000 populations. ${ }^{11}$ However, the difference in prevalence of Lymphoma is not much affected by gender $(8.4 / 100000$ in male versus $6.5 / 100000$ in female population ${ }^{4}$ ). In Pakistan, breast cancer patients usually present during advanced stages of disease due to low awareness about the illness and socio-economic hurdles. A study conducted in Karachi reported that most of the patients presented during stage 2 and 3 of the disease $^{12}$. In our experience at PIMS Hospital Islamabad, most of the patients presented during stage 3. Similarly, Lymphoma patients in Pakistan mostly present at stage 2 and $3{ }^{13}$

In the present study both LS and BCS groups had good quality of life post treatment in physical and psychological parameters, however the Breast Cancer Survivor (BCS) group reported lower mean values as compared to Lymphoma Survivors (LS) group in Physical health parameters. There was worse score in parameters like fatigue, body aches and menstrual and fertility issues in BCS group as compared to LS group. Due to the unique characteristics of breast cancer and lymphoma, disparity in the sample size with respect to gender was seen.
A study conducted at Agha Khan University Karachi, Pakistan reported that post chemotherapy cancer patients reported low QOL due to issues in physical and emotional wellbeing. ${ }^{14}$ However, this is in contrast to a study conducted at Combined Military Hospital, Rawalpindi ${ }^{15}$ where breast cancer patients following mastectomy, described good QOL perceptions in physical wellbeing parameters. Raymond et al. ${ }^{16}$ reported that post mastectomy pain is present in almost $20-50 \%$ of the patients, which severely deteriorated the QOL of these individuals and its risk factors included physical, genetic as well as psychological factors. In a study conducted in University of California, ${ }^{17}$ at least $25 \%$ of the patients reported postoperative pain after mastectomy and preoperative depression and anxiety was associated with higher levels of pain. Costa et al. from Brazil reported that more than $70 \%$ breast cancer patients reported pain, which directly affected the physical health and functional capacity of these individuals, resulting in increased morbidity. ${ }^{18}$ Depression and post-traumatic stress disorder also resulted in low physical component of QOL amongst Lymphoma survivors as reported by Compaci et al. ${ }^{19}$

Although there was insignificant difference of overall psychological impact on QOL in both groups in our study, the results showed breast cancer survivors had more concerns about their change of 
appearance and self-image, post-surgery and postchemotherapy. Higher distress of disease and fear of recurrence was also noted by the researchers in this study among BCS group as compared to Lymphoma group. Sema et al. reported that women who had undergone mastectomy reportedly gave negative statements about their body image and felt they were half of themselves. ${ }^{20}$ Sophia et al. reported that there was better physical health but worse psychological health in young lymphoma survivors after controlling demographic co-relations. ${ }^{21}$ Poor psychological health amongst Lymphoma survivors was associated with older age, higher co-morbidities and low education status. ${ }^{22-24}$

In our study, the patients who had undergone mastectomy reported feeling decreased sexuality. This is consistent with the findings reported by Jose and Maria with participants feeling that they had mutilated bodies with decreased femininity resulting in low self-esteem, less physical attractiveness and inability to keep social relationships. ${ }^{25}$ Similar concerns were reported from Turkey, where respondents felt that mastectomy negatively affected their relationship with their partners and their social interactions were also affected. ${ }^{20}$

The main limitation of our study was that we assessed the QOL of cancer survivors at least 6 months after their treatment. However, a longer duration of follow-up is required to study long term psychosomatic and social effects of treatment in both BCS and LS groups

\section{Conclusion}

Both lymphoma and breast cancer negatively affect the quality of life of the patients especially the psychological, social and spiritual wellbeing. However, surgical intervention led to considerable worsening of quality of life with respect to physical and social wellbeing and increased distress of illness and fear factor.

\section{Recommendations}

Psychological and Social support groups as well as rigorous follow up for both physical and psychological illnesses should be considered in cancer survivors. Breast reconstruction surgery should be made available in all breast cancer clinics and centers so that the social and psychological impact of the disease and its treatment can be minimized.

\section{Acknowledgment}

Authors are thankful to Ms. Mahwish Raza (Medical Affairs, Getz Pharma) for providing assistance in statistical analysis and formatting manuscript.

\section{References}

1. Index C. Cancer Index Pakistan. Cancerindex.org. 2020. Available from: http://www.cancerindex.org/Pakistan

2. Van Leeuwen M, Husson O, Alberti P, Arraras J, Chinot $\mathrm{O}$, Costantini $A$, et al. Understanding the quality of life (QOL) issues in survivors of cancer: towards the development of an EORTC QOL cancer survivorship questionnaire. Health Qual Life Outcomes. 2018; 16(1): 114. Doi: 10.1186/s12955-018-0920-0

3. Ayanian JZ, Jacobsen PB. Enhancing research on cancer survivors. J Clin Oncol. 2006; 24(32): 5149-53. Doi: 10.1200/JCO.2006.06.7207

4. Bhurgri $Y$, Pervez S, Bhurgri S, Faridi N, Usman A, Kazi LA et al. Increasing incidence of Non-Hodgkin's lymphoma in Karachi, 1995-2002. Asian Pac J Cancer Prev. 2005; 6(3): 364-9. PMID: 16236001

5. Khalfan Z, Edgar K, Ali A Z, Sadruddin S. Breast Cancer in Pakistani females. Journal on Nursing. 2014; 4(2): 13-17. Doi: 10.26634/jnur.4.2.2771

6. NCCN clinical practice guidelines in Oncology (NCCN guidelines) for Hodgkin Lymphoma. Version 2.2019.

7. Sharma GN, Dave R, Sanadya J, Sharma P, Sharma KK. Various types and management of breast cancer: an overview. J Adv Pharm Technol Res. 2010; 1(2):10926. PMID: 22247839

8. Valdes-Stauber J, Vietz E, Kilian R. The impact of clinical conditions and social factors on the psychological distress of cancer patients: an explorative study at a consultation and liaison service in a rural general hospital. BMC Psychiatry. 2013; 13(1). 
9. Fujisawa D, Hagiwara N. Cancer Stigma and its Health Consequences. Current Breast Cancer Reports. 2015;7(3):143-150.

10. Erturhan Turk K, Yilmaz M. The Effect on Quality of Life and Body Image of Mastectomy Among Breast Cancer Survivors. European Journal of Breast Health. 2018;:205-210.

11. Gill M, Kayani N, Khan M, Hasan S. Breast Diseases in Males - A Morphological Review of 150 Cases. JPMA. 2000; 50(6): 177-9. PMID: 10979622

12. Soomro R, Faridi S, Khurshaidi N, Zahid N, Mamshad I. Age and stage of breast cancer in Pakistan: An experience at a tertiary care center. JPMA. 2018; 68(11): 1682-85. PMID: 30410149

13. Mumtaz T, Roohi N, Akhtar M. Incidence and Clinical Manifestation of Lymphoma in Central Punjab. Pakistan J Zool. 2012; 44(5): 1367-72.

14. Chagani P, Parpio Y, Gul R, Jabbar AA. Quality of Life and Its Determinants in Adult Cancer Patients Undergoing Chemotherapy Treatment in Pakistan. Asia Pac J Oncol Nurs. 2017; 4(2): 140-146. Doi:10.4103/2347-5625.204499

15. Ozair DN, Kamran DJ, Asim DS, Fatima DA, Hisam DA, Ujala DM. Quality of life among post-surgical patients of breast cancer visiting a tertiary care hospital in Rawalpindi. PAFMJ.2019; 69(SUPPL 2): S303-9.

16. Tait RC, Zoberi K, Ferguson M, et al. Persistent PostMastectomy Pain: Risk Factors and Current Approaches to Treatment. J Pain. 2018; 19(12): 13671383. Doi: 10.1016/j.jpain.2018.06.002

17. Miaskowski C, Cooper B, Paul SM, West C, Langford $D$, Levine JD, et al. Identification of patient subgroups and risk factors for persistent breast pain following breast cancer surgery. J Pain. 2012; 13(12): 11721187. Doi: 10.1016/j.jpain.2012.09.013
18. Costa W, Eleutério Jr. J, Giraldo P, Gonçalves A. Quality of life in breast cancer survivors. Revista da Associação Médica Brasileira. 2017; 63(7): 583-89. Doi: 10.1590/1806-9282.63.07.583

19. Compaci G, Conte C, Oberic L, Ysebaert L, Laurent G, Despas F. Sustained degradation of quality of life in a subgroup of lymphoma survivors: a two-year prospective survey. BMC Cancer. 2019; 19(1): 1178. Doi: 10.1186/s12885-019-6337-2

20. Koçan S, Gürsoy A. Body Image of Women with Breast Cancer After Mastectomy: A Qualitative Research. J Breast Health. 2016; 12(4): 145-50. Doi:10.5152/tjbh.2016.2913

21. Smith S, Crespi C, Petersen L, Zimmerman S, Ganz P. The impact of cancer and quality of life for posttreatment non-Hodgkin lymphoma survivors. PsychoOncology. 2010; 19(12): 1259-67. Doi: 10.1002/pon.1684

22. Ng D, Leong Y, Gan G. Quality of life amongst lymphoma survivors in a developing country. Supportive Care Cancer. 2016; 24(12): 5015-23. Doi: 10.1007/s00520-016-3364-2

23. Drost FM, Mols F, Kaal SEJ, Stevens WBC, van der Graaf WTA, Prins JB, et al. Psychological impact of lymphoma on adolescents and young adults: not a matter of black or white. J Cancer Surviv. 2016; 10(4): 726-35. Doi:10.1007/s11764-016-0518-7

24. Smith S, Mayer D, Zimmerman S, Williams C, Benecha $H$, Ganz $P$, et al. Quality of Life Among Long-Term Survivors of Non-Hodgkin Lymphoma: A Follow-Up Study. J Clin Oncol. 2013; 31(2): 272-279. Doi: 10.1200/JCO.2011.40.6249

25. Arroyo J, López M. Psychological Problems Derived from Mastectomy: A Qualitative Study. Int J Surg Oncol. 2011; 1-8. Doi: 10.1155/2011/132461 